\title{
ON A PROBLEM OF BARNES AND DUNCAN
}

\author{
by R. G. MCLEAN
}

(Received 5th February 1990)

Consider the free monoid on a non-empty set $P$, and let $R$ be the quotient monoid determined by the relations:

$$
p^{2}=p \quad \forall p \in P .
$$

Let $R$ have its natural involution * in which each element of $P$ is Hermitian. We show that the Banach *-algebra $\ell^{1}(R)$ has a separating family of finite dimensional *-representations and consequently is *-semisimple. This generalizes a result of B. A. Barnes and J. Duncan (J. Funct. Anal. 18 (1975), 96-113.) dealing with the case where $P$ has two elements.

1980 Mathematics subject classification (1985 Revision): 43 A65

Consider the free monoid on a non-empty set $P$, and let $R$ be the quotient monoid determined by the relations:

$$
p^{2}=p \quad \forall p \in P
$$

We equip $R$ with its natural involution $*$ in which each element of $P$ is Hermitian. When $P$ contains exactly two elements Barnes and Duncan [2] have shown that the Banach *-algebra $\ell^{1}(R)$ has a separating family of finite dimensional *-representations. We show that this result is in fact true for an arbitrary $P$. It follows that $\ell^{1}(R)$ is *-semisimple.

Let $S$ be a monoid i.e. a semigroup with an identity element 1. By a representation $\pi$ of $S$ we shall mean a bounded map $\pi$ from $S$ into the set of all bounded linear operators on a (real or complex) Hilbert space $H$ such that $\pi(1)$ is the identity operator and $\pi(x y)=\pi(x) \pi(y)$ for all $x, y \in S$. Each representation of $S$ has a unique extension to a representation of the Banach algebra $\ell^{1}(S)$. Tensor products and direct sums may be formed in a similar way to those of group representations.

Definition. A representation $\pi$ of a monoid $S$ on a Hilbert space $H$ will be called formally real is there is an orthonormal basis $\left\{e_{i} \mid i \in I\right\}$ of $H$ for which $\left\langle\pi(x) e_{s} \mid e_{r}\right\rangle$ is real for all $x \in S$ and all $r, s \in I$.

It is known that if the *-representations of $S$ are separating then the Banach *-algebra $\ell^{1}(s)$ is *-semisimple. This follows from Theorem 3.4 of [1] (a direct proof may be found 
in [3]). The following lemma shows that under suitable conditions a separating set of representations of $S$ gives rise to a separating set of representations of $\ell^{1}(S)$.

Lemma. Let $\mathscr{Q}$ be a set of formally real representations of a monoid $S$ and let $\mathscr{R}$ be the set of representations of the Banach *-algebra $\ell^{1}(S)$ consisting of the one dimensional identity representation together with all extensions of finite tensor products of members of $\mathscr{Q}$, If 2 separates points of $S$, then $\mathscr{R}$ separates points of $\ell^{1}(S)$.

Proof. Let $\mathscr{A}$ be the set of all functions $f: S \rightarrow \mathbb{F}$ with

$$
f(x)=\langle\pi(x) \phi \mid \psi\rangle \quad \forall x \in S
$$

for some representation $\pi$ of $S$ on a Hilbert space $H$ and some vectors $\phi, \psi \in H$, where $\pi$ is a finite direct sum of representations from $\mathscr{R}$. Then $\mathscr{A}$ is a self-conjugate unital subalgebra of $\ell^{\infty}(S)$ which separates points of $S$ so by Theorem 1 of [3], if $f$ is a non-zero element of $\ell^{1}(S)$, then there is a $g \in \mathscr{A}$ with

$$
\sum_{x \in S} f(x) g(x) \neq 0
$$

It follows that there is a $\pi \in \mathscr{R}$ with $\pi(f) \neq 0$.

Theorem. The Banach *-algebra $\ell^{1}(R)$ has a separating family of finite dimensional *-representations.

Proof. Let $T$ denote the quotient of the free monoid on $\{u, v\}$ determined by the equations $u^{2}=u$ and $v^{2}=v$; then $T$ has an injective two dimensional *-representation. For if $\pi$ is the *-representation, considered in [2], defined by

$$
\pi(u)=\left[\begin{array}{ll}
1 & 0 \\
0 & 0
\end{array}\right], \quad \pi(v)=\frac{1}{2}\left[\begin{array}{ll}
1 & 1 \\
1 & 1
\end{array}\right]
$$

then for any $n \in \mathbb{N}$ :

$$
\begin{aligned}
& \pi\left((u v)^{n}\right)=2^{-n}\left[\begin{array}{ll}
1 & 1 \\
0 & 0
\end{array}\right], \quad \pi\left((u v)^{n-1} u\right)=2^{-(n-1)}\left[\begin{array}{ll}
1 & 0 \\
0 & 0
\end{array}\right], \\
& \pi\left((v u)^{n}\right)=2^{-n}\left[\begin{array}{ll}
1 & 0 \\
1 & 0
\end{array}\right], \quad \pi\left((v u)^{n-1} v\right)=2^{-(n-1)}\left[\begin{array}{ll}
1 & 1 \\
1 & 1
\end{array}\right],
\end{aligned}
$$

and it follows that $\pi$ is injective. 
For each ordered pair $(p, q) \in P^{2}$ with $p \neq q$, let $\psi_{(p, q)}$ be the surjective *-morphism from $R$ to $T$ determined by

$$
\psi_{(p, q)}(w)= \begin{cases}u & \text { if } w=p \\ v & \text { if } w=q \\ 1 & \text { if } w=1 \text { or } w \in P \backslash\{u, v\}\end{cases}
$$

and let $\pi_{(p, q)}$ be the *-representation $\pi \circ \psi_{(p, q)}$. We show that the set $2=\left\{\pi_{(p, q)} \mid\right.$ $p \in P, q \in P, p \neq q\}$ separates points of $R$.

Each element of $R \backslash\{1\}$ may be written as a word in the alphabet $P$ in which no two adjacent letters are equal. Let $x$ and $y$ be distinct elements of $R$. If one of these is the identity let $p$ be the first letter of the other, then $\psi_{(p, q)}(x) \neq \psi_{(p, q)}(y)$ for any $q \in P$.

If one word, say $x$, is a prefix of the other, then there are $p, q \in P$ and $a, b \in R$ with

$$
x=a p \quad \text { and } \quad y=a p q b
$$

and now

$$
\psi_{(p, q)}(x) \neq \psi_{(p, q)}(y)
$$

Otherwise let $p, q \in P$ be the first two letters in which the words $x$ and $y$ differ; then clearly

$$
\psi_{(p, q)}(x) \neq \psi_{(p, q)}(y)
$$

Since $\pi$ is injective it follows that $\mathcal{Q}$ separates points of $R$. Also since $\pi$ is two dimensional it follows from the lemma that $\ell^{1}(R)$ has a separating family of finite dimensional *-representations.

Acknowledgement. This work was carried out at Queen's University, Kingston, Ontario, Canada. I wish to thank the Leverhulme Trust for financial support and $\mathrm{H}$. Kummer for his hospitality.

\section{REFERENCES}

1. M. Lashrarizadeh Bami, Representations of foundation semigroups and their algebras, Canad. J. Math. 37 (1985), 29-47.

2. B. A. Barnes and J. Duncan, The Banach algebra $\ell^{1}(S)$, J. Funct. Anal. 18 (1975), 96-113.

3. R. G. McLean and H. Kummer, Representations of the Banach algebra $\ell^{1}(S)$, Semigroup Forum 37 (1988), 119-122.

Department of Mathematics

The City University

Northampton SQuare

LONDON ECIV OHB

UNITED KINGDOM 